\title{
Quantifying the Significance of the Magnetic Field from Large-Scale Cloud to Collapsing Core: Self-Similarity, Mass-to-Flux Ratio and Star Formation Efficiency
}

\author{
Patrick M. Koch ${ }^{1}$, Ya-Wen Tang ${ }^{2,3}$ \& Paul T. P. Ho ${ }^{1,4}$ \\ pmkoch@asiaa.sinica.edu.tw
}

\begin{abstract}
Dust polarization observational results are analyzed for the high-mass star formation region W51 from the largest parent cloud $(\sim 2 \mathrm{pc}, \mathrm{JCMT})$ to the large-scale envelope ( $\sim 0.5 \mathrm{pc}, \mathrm{BIMA})$ down to the collapsing core e $2(\sim 60 \mathrm{mpc}$, SMA). Magnetic field and dust emission gradient orientations reveal a correlation which becomes increasingly more tight with higher resolution. The previously developed polarization - intensity gradient method (Koch et al. 2012) is applied in order to quantify the magnetic field significance. This technique provides a way to estimate the local magnetic field force compared to gravity without the need of any mass or field strength measurements, solely making use of measured angles which reflect the geometrical imprint of the various forces. All three data sets clearly show regions with distinct features in the field-to-gravity force ratio. Azimuthally averaged radial profiles of this force ratio reveal a transition from a field dominance at larger distances to a gravity dominance closer to the emission peaks. Normalizing these profiles to a characteristic core scale points toward self-similarity. Furthermore, the polarization intensity-gradient method is linked to the mass-to-flux ratio, providing a new approach to estimate the latter one without mass and field strength inputs. A transition from a magnetically supercritical to a subcritical state as a function of distance from the emission peak is found for the e 2 core. Finally, based on the measured radius-dependent field-to-gravity force ratio we derive a modified star formation efficiency with a diluted gravity force. Compared to a standard (free-fall) efficiency, the observed field is capable of reducing the efficiency down to $10 \%$ or less.
\end{abstract}

\footnotetext{
${ }^{1}$ Academia Sinica, Institute of Astronomy and Astrophysics, Taipei, Taiwan

${ }^{2}$ Université de Bordeaux, Observatoire Aquitain des Sciences de l'Univers, 2 rue de l'Observatoire, BP 89, F-33271 Floirac Cedex, France

${ }^{3}$ CNRS, UMR 5804, Laboratoire d'Astrophysique de Bordeaux, 2 rue de l'Observatoire, BP 89, F-33271 Floirac Cedex, France

${ }^{4}$ Harvard-Smithsonian Center for Astrophysics, 60 Garden Street, Cambridge, MA 02138, USA
} 
Subject headings: ISM: clouds — ISM: magnetic fields, polarization — ISM: individual (W51, W51 e2) — Methods: polarization

\section{Introduction}

The magnetic field is being recognized as a crucial component in the star formation process. Evidence for its presence and significance is growing with the advance of an increasing number of instruments capable of providing high-quality polarization observations. To date, various observations - based on different emission or absorption mechanisms over a range of wavelengths - cover scales from a few pc down to mpc in molecular clouds. Nevertheless, the precise role of the magnetic field and its interplay with, e.g. turbulence and gravity, remain a debated topic in the current literature. In order to make further progress, knowledge of both the field morphology and the field strength needs to be combined. It is unfortunate that many of the currently available observational techniques can only address one or the other of these field properties. Whereas dust polarization reveals a plane of sky projected magnetic field orientation and morphology (e.g., Hildebrand 1988), it does not provide any information about the magnetic field strength. On the other hand, Zeeman splitting in spectral lines (e.g., Crutcher et al. 2009) gives a line-of-sight field strength, but typically the field morphology is not recovered due to the only isolated detections. An exception might be the recent works by Surcis et al. (2011) and Vlemmings et al. (2011), where the morphology starts to become visible based on several tens of maser detections.

Besides the intrinsic shortcomings of the observing techniques mentioned above, analysis tools and interpretation of polarization data are still in a developmental stage. Consequently, the benefit of the additional polarization information beyond its mere detection and imaging purpose is often not very obvious. With the growing number of high-quality polarization observations from various instruments, it is, thus, paramount to investigate new methods and strategies to further explore and reveal the physics hidden in polarization data. In order to make further progress, efforts on several fronts are needed and eventually must be combined. On one hand, magneto-hydrodynamic simulations producing synthetic observational maps can characterize generic features and provide guidelines for data interpretation (e.g., Nakamura \& Li 2011; Falceta-Gonçalves et al. 2008; Li \& Nakamura 2004; Allen et al. 2003). It is further desirable to include radiative transfer modeling in this approach. On the other hand, methods and techniques inspired by observed data can lead to new unexpected insights in a phenomenological way. Such recent approaches are, e.g., the polarization dispersion function used to trace the relative turbu-

lence level (Houde et al. 2009; Hildebrand et al. 2009) and the ambipolar diffusion scale isolated by comparing coexisting ion and molecular line spectra (Hezareh et al. 2011; Li \& Houde 2008). Here, we are further exploring the recently developed polarization - intensity gradient method 
(Koch et al. 2012). This technique leads to a local position-dependent measurement of the magnetic field strength, and it additionally provides an estimate of the local field-to-gravity force ratio in a model-independent way.

We are applying our method to a set of dust emission polarization data in the sub-millimeter regime, where the dust grains are thought to be aligned with their shorter axis parallel to the magnetic field lines due to radiative torques (Draine \& Weingartner 1996, 1997; Lazarian 2000). The emitted light, therefore, appears to be polarized perpendicular to the field lines (e.g., Hildebrand 1988). Dust polarization observations are now being routinely carried out with the Submillimeter Array (SMA) (Tang et al. 2012; in preparation; Tang et al. (2010, 2009b.a); Rao et al. (2009); Girart et al. (2009, 2006)). This study is part of the program on the SMA 1 (Ho et al. 2004) to investigate the structure of the magnetic field from large to small scales.

The paper is organized as follows. Focusing on the magnetic field detections in the W51 star formation region, Section 2 describes the relevant polarization observations from the JCMT, BIMA and the SMA. The key results of the polarization - intensity gradient method (Koch et al. 2012) which serve as a starting point for this work here - are summarized in Section 3. Section 4 starts with pointing out the correlation between the magnetic field and intensity gradient orientations, and then presents our results on the magnetic field significance. Implications of our findings for the mass-to-flux ratio and the star formation efficiency are discussed in Section 5, A summary and conclusion are given in Section 6 ,

\section{Observations and Source Description}

The W51A2 cloud is one of the most active and luminous high mass star formation sites in the Galaxy. Located at a distance of $\sim 7 \mathrm{kpc}$ (Genzel et al. 1981), $1^{\prime \prime}$ is equivalent to $\sim 0.03 \mathrm{pc}$. Chrysostomou et al. (2002) measured the polarization at $850 \mu \mathrm{m}$ with SCUBA on the JCMT across the molecular cloud at a scale of 5 pc with a binned resolution $\theta \approx 9$ !!3. Their observation encompasses two cores with polarized emission detected both in the cores and the region in between them. At this scale, the polarization appears to be organized but with a morphology that changes from the dense cores to the surrounding more diffuse areas. Lai et al. (2001) reported a higher angular resolution $\left(3^{\prime \prime}\right)$ polarization map at $1.3 \mathrm{~mm}$ obtained with BIMA, which resolved out large-scale structures. In contrast to the larger scale JCMT map, the polarization appears to be uniform across

\footnotetext{
${ }^{1}$ The Submillimeter Array is a joint project between the Smithsonian Astrophysical Observatory and the Academia Sinica Institute of Astronomy and Astrophysics, and is funded by the Smithsonian Institution and the Academia Sinica.

2 The source names W51A (G49.5-0.4) or W51 are used synonymously in the following sections.
} 
the envelope at a scale of $0.5 \mathrm{pc}$, resolving the eastern core in the JCMT map into the sources W51 $\mathrm{e} 2$ and e8. W51 e 2 is one of the strongest $\mathrm{mm} / \mathrm{submm}$ continuum sources in the W51A region. In the highest angular resolution map obtained with the SMA at $870 \mu \mathrm{m}$ with $\theta \approx 0.7$, the polarization patterns appear to be pinched in e 2 and also possibly in e8 (Tang et al. 2009b). In particular, the structure detected in the collapsing core e 2 reveals hourglass-like feature 3 . These data currently provide the highest angular resolution information on the morphology of the magnetic field in the plane of sky obtained with the emitted polarized light in the W51A star formation site. A statistical analysis based on a polarization structure function (of second order) shows a turbulent to mean magnetic field ratio which decreases from the larger (BIMA) to the smaller (SMA) scales from $\sim 1.2$ to $\sim 0.7$ (Koch et al. 2010), possibly demonstrating that the role of magnetic field and turbulence evolves with scale. Collapse signatures in W51 have been reported for various molecules in Rudolph et al. (1990); Ho \& Young (1996); Zhang \& Ho (1997); Young et al. (1998); Zhang et al. (1998); Solins et al. (2004).

Figure 1 reproduces the dust continuum Stokes I maps from the JCMT, BIMA and the SMA observations 4 , with their original (phase) centers at Right Ascension (J2000) $\alpha=19^{\mathrm{h}} 23^{\mathrm{m}} 39^{\mathrm{s}} .0$, Declination $(\mathrm{J} 2000) \delta=14^{\circ} 31^{\prime} 08^{\prime \prime} .00 ; \alpha=19^{\mathrm{h}} 23^{\mathrm{m}} 44^{\mathrm{s}} .2, \delta=14^{\circ} 30^{\prime} 33^{\prime \prime} .4$ and $\alpha=19^{\mathrm{h}} 23^{\mathrm{m}} 43^{\mathrm{s}} .95, \delta=14^{\circ} 30^{\prime} 34^{\prime \prime} 00$. Overlaid in red are the magnetic field segments, rotated by $90^{\circ}$ with respect to the originally detected polarization orientations. Typical measurement uncertainties of individual position angles are in the range of $5^{\circ}$ to $10^{\circ}$ and $3^{\circ}$ to $9^{\circ}$ for the SMA and for BIMA, respectively. The median uncertainty in the JCMT data is about $5^{\circ}$, with a few outliers between $30^{\circ}$ and $40^{\circ}$. Additionally shown are the intensity gradient orientations in blue, which are relevant for the further analysis in the following sections.

We remark that W51A was also observed with the $350 \mu \mathrm{m}$ polarimeter Hertz at the Caltech Submillimeter Observatory with a resolution of $\sim 20^{\prime \prime}$ (Dotson et al. 2010). For our purposes here, we have found these data to be in agreement with and equivalent to the JCMT observation. They are, thus, not further discussed here.

\section{A Model Independent Approach: the Polarization - Intensity Gradient Method}

Various forces are interacting in molecular clouds. Maps of observed dust emission are reflecting the overall result of gravity, pressure and magnetic field forces, and possible additional

\footnotetext{
${ }^{3}$ Regardless of the different physical scales in the JCMT, BIMA and the SMA observations, areas in a any map identified by clear emission peaks are denoted as cores, here and in the following sections.

${ }^{4}$ The reduced JCMT data are available at http://cdsarc.u-strasbg.fr/viz-bin/qcat?J/A+A/
} 
constituents. Dust emission and magnetic field morphologies are left with a geometrical imprint by the combined effect of all these forces. Interestingly, polarization orientations are often observed to be close to tangential to dust emission intensity contours. Therefore, magnetic field and intensity gradient directions show a correlation, where the difference $\delta$ in their orientations can be linked to the magnetic field strength (Figure 3 in Koch et al. (2012)). In this method, various components in the ideal magneto-hydrodynamic (MHD) force equation are identified in dust polarization and Stokes $I$ maps. In particular, a change in emission intensity (gradient) is assumed to be the result of the transport of matter driven by the combination of all the forces in the MHD equation. Adopting this, it then follows that the gradient in the dust emission Stokes $I$ intensity defines the resulting direction of motion in the MHD force equation. A force triangle, where the vector sum of all forces is set equal to the intensity gradient, can then be constructed (Figure 3 in Koch et al. (2012)). As a result, the total magnetic field strength as a function of position in a map can be calculated as:

$$
B=\sqrt{\frac{\sin \psi}{\sin \alpha}(\nabla P+\rho \nabla \phi) 4 \pi R},
$$

where the angle $\psi$ is the difference in orientations between the gravitational pull and the intensity gradient, and the angle $\alpha$ is the difference between the polarization and the intensity gradient orientations. $\rho \nabla \phi$ and $\nabla P$ are the gravitational pull and the pressure gradient, respectively. $R$ is the magnetic field radius. Generally, all variables are functions of positions in a map. In the case of W51 e2, when neglecting the pressure gradient, the field strengths vary between $\sim 2 \mathrm{mG}$ and $\sim 20 \mathrm{mG}$ with a radial profile $B(r) \sim r^{-1 / 2}$ (Koch et al. 2012). The field strength averaged over the e 2 core is $\sim 7.7 \mathrm{mG}$.

In order to determine the field strength $B$ in Equation (1), the mass (gravitational potential $\phi$ ) and the pressure gradient, if significant, need to be known. Contrary to this absolute field strength measure, the relative importance of the field compared to the other forces is directly imprinted in the field and intensity morphologies. With the magnetic field tension force $F_{B}=\frac{B^{2}}{4 \pi R}$ and the gravitational and pressure forces $\left|F_{G}+F_{P}\right|=|\rho \nabla \phi+\nabla P|$, Equation (1) is rewritten as:

$$
\left(\frac{F_{B}}{\left|F_{G}+F_{P}\right|}\right)_{\text {local }}=\left(\frac{\sin \psi}{\sin \alpha}\right)_{\text {local }} \equiv \Sigma_{B},
$$

where we have introduced $\Sigma_{B}$ to define the field significance. The polarization - intensity gradient method, thus, provides a way to estimate the local magnetic field significance relative to other forces in a model-independent way. It is based on a generally valid (ideal) MHD equation, but it is independent of any molecular cloud/core models. Furthermore, this technique to extract the field significance is free of any necessity of mass and field strength measurements. The ratio in Equation (2) is solely based on measured angles which reflect the geometrical imprint of the various forces. Consequently, this also means that molecular clouds with accordingly scaled masses and field 
strengths can show identical morphologies. This self-similar (or scale-free) property is lifted when calculating the field strength $B$ for a particular cloud mass in Equation (1).

In any case, the angle factor $\frac{\sin \psi}{\sin \alpha}=\Sigma_{B}$ provides a quantitative criterion as to whether the magnetic field can prevent an area in a molecular cloud from gravitational collapse $\left(\Sigma_{B}>1\right)$ or not $\left(\Sigma_{B}<1\right)$. As further demonstrated in Koch et al. (2012), $\Sigma_{B}$ is only minimally or even not at all affected by projection effects.

\section{Results}

\subsection{Magnetic Field - Intensity Gradient Correlation}

The dust continuum Stokes I maps of the JCMT, BIMA and the SMA are shown in the left column panels in Figure 1. In this sequence of subsequently higher resolution maps, the JCMT main core $\left(\sim 40^{\prime \prime}\right.$ in size $)$ is resolved into two cores $\left(\sim 8^{\prime \prime}\right)$ in the BIMA observation, where the Northern core is further resolved into W51 e2 $\left(\sim 2^{\prime \prime}\right)$ in the SMA map. Overlaid on the dust continuum maps are the magnetic field segments (red) and the intensity gradient segments (blue). For completeness, the SMA result for W51 e2 is reproduced from Koch et al. (2012). A tight correlation between magnetic field position angle (P.A.) and the corresponding intensity gradient P.A. (angle $\delta<\pi / 2$ in Figure 3 in Koch et al. (2012)) is obvious for many of the pairs. The correlation coefficients - in the definition of Pearson's linear correlation coefficient - are 0.71, 0.72 and 0.95 for the JCMT, BIMA and the SMA data, respectively. Thus, there is seemingly a trend for an increasing alignment between magnetic field and intensity gradient orientations with smaller scales, with the tightest correlation found for the collapsing core. This correlation is being investigated in a separate work on a larger data sample (Koch et al. 2012; in preparation). It suffices to mention here that the above correlation can possibly serve as an indicator for the role of the magnetic field through the evolutionary stages of a molecular cloud.

\subsection{Relative Magnetic Field Significance from Large to Small Scales}

Maps of the magnetic field to gravitational force ratio, $\Sigma_{B}=\frac{\sin \psi}{\sin \alpha}$, are displayed in the right column panels in Figure 1. The pressure gradient force, $\nabla P$, typically being small compared to gravity, is omitted here. For the JCMT and BIMA data, two gravity centers at the two emission peaks in each map are assumed in order to calculate $\psi$. The angle $\psi$ is then simply measured in between the intensity gradient direction (left column panels in Figure 1) and the gravity center direction. For W51 e2 (SMA data), a single gravity center at the emission peak in the SMA map is 
adopted. The angle $\alpha=\pi / 2-\delta$, where $\delta$ is the difference in between the intensity gradient and the magnetic field orientations, is deduced from the left column panels in Figure 1. All three maps originating from three different instruments, one single dish and two interferometers - show clear differences in the ratios between core regions and areas in between the cores or further away. With the largest mapping area, the JCMT observation reveals very distinct features. With the exception of only two segments in the eastern core, both core regions show ratios below 0.5. In between the two cores the ratios reveal peaks in between 1.5 and $\sim 5$, with large stretches in the northeastsouthwest direction larger than one. The BIMA observation is rather restricted to the cores, with an area in the northern core without polarization detection. Nevertheless, both cores clearly show ratios below one, except one segment in the southern core. The four segments in east-west direction (around y-offset $\sim-4$ ) reveal ratios larger or around one. Though limited to a few segments only, this still points toward a trend of increased ratios outside the immediate core areas. The detection by the SMA mostly resolves polarization features in the e 2 core. The ratio averaged over the core is $\sim 0.2$. The north-west extension shows two segments with a ratio larger than one, and an average of about 0.8 . If assuming an additional new core being formed here, the ratios get reduced to about 0.5 . In any case, the main collapsing core very clearly shows ratios below one, whereas the more distant north-west extension shows larger values. We remark that the total outflow mass in e2 $\left(\sim 1.4 M_{\odot}\right.$, Shi et al. (2010) ) is negligibly small compared to the total core mass of about $220 M_{\odot}$ (Tang et al. 2009b). Additionally, with a dust-to-gas ratio of about 1 to 100, the outflow is unlikely to be detected in the dust continuum with the SMA sensitivity. Consequently, we do not expect the magnetic field and the dust continuum Stokes $I$ morphologies to be significantly affected by outflows. Our analysis, therefore, still quantifies the field to gravitational force ratio. The SMA e8 core is not further analyzed here because only a few polarization segments are detected (Tang et al. 2009b).

In summary, the cores in the JCMT, BIMA and SMA data have average ratios of $0.33,0.45$ (east, west), 0.38, 0.49 (north, south) and $\sim 0.2$ (main core), respectively. The other areas reveal significantly and systematically larger ratios $(\gtrsim 1)$. Despite probing three very different physical scales, the three data sets equally reveal a minor role of the magnetic field, $F_{B}<F_{G}$, in the center regions, and an increasingly more significant field, $F_{B} \gtrsim F_{G}$, at larger distances.

The distinct features of the field-to-gravity force ratio in the right panels in Figure 1 are further analyzed in the following. Azimuthally averaged radial profiles, binned at half of the beam resolution, are displayed in Figure 2. The profiles are centered at the dust continuum peaks. From the JCMT data, the main (eastern) peak covering the BIMA observation and the SMA e2 core is selected. On the next smaller scale, the BIMA southern core is chosen because its force ratio in the center has a smaller uncertainty than the northern core. As already manifest in the right panels in Figure 1, a clear difference between center and outer regions is seen. Both the JCMT and SMA profiles show ratios across the center $(\sim 0.3$ for JCMT and $\sim 0.2$ for SMA) with relatively little 
variations. This is followed by a rather abrupt increase over a short distance (one bin) by a factor of $\sim 2$ (JCMT) and $\sim 4$ (SMA). A plateau, with ratios $\sim 0.8$ for both JCMT and SMA, then extends over distances comparable to or larger than the center regions. The JCMT data, with the largest mapped area, then show another abrupt change to a ratio beyond one. No polarized emission is detected for W51 e2 at larger distances. The case of BIMA is less clear, but still shows values in the center of $\sim 0.1$ and $\sim 0.6$, followed by a plateau around 0.6 and an increase to larger than one at the largest distances. For comparison, azimuthally averaged emission intensity profiles are also shown.

For completeness, profiles for the JCMT western and the BIMA northern core are shown in Figure 3. The result for the very central region in the BIMA northern core is less conclusive because only a single value around 0.7 with a $\pm 50 \%$ error is available for the force ratio. Nevertheless, values are clearly below one in the core region with a trend to grow at larger distances up to about 0.9 . Similarly to the eastern core, the western core in the JCMT data shows ratios around 0.2 to 0.6 up to about $20^{\prime \prime}$ in distance. Further away from the peak, ratios grow up to about 5 with some oscillatory behavior remaining larger than one. Thus, despite being less pronounced, the two additional cores here also reveal a transition in their force ratios between central and more distant ratios. In particular, in the overlapping region between the BIMA northern core and the SMA e2 core, the ratios show similar trends regardless of the different field morphologies (hourglass-like for SMA, more uniform for BIMA).

Errors in the field-to-gravity force ratio are calculated by propagating the measurement uncertainties $\Delta \psi$ and $\Delta \alpha$ through Equation (2). The measured magnetic field P.A. uncertainty, in the range of a few degrees to $\sim 10^{\circ}$, is assumed for $\Delta \alpha$. A typical uncertainty of $3^{\circ}-5^{\circ}$ in $\Delta \psi$ results after interpolation when calculating the intensity gradients. This leads to errors for individual ratios in Figure 2 of $\sim \pm 4 \%$ to $\pm 19 \%, \sim \pm 4 \%$ to $\pm 40 \%$ and $\sim \pm 2 \%$ to $\pm 20 \%$ (with a single outlier at $\pm 60 \%$ ) for the SMA, BIMA and the JCMT data, respectively. After averaging in each bin, the errors are typically reduced due to the sample variance factor, resulting in average errors of $\sim \pm 10 \%$ or less. Average errors of $\sim \pm 20 \%$ remain for two bins in the JCMT data. A $10 \%$ measurement uncertainty is conservatively estimated for the emission intensity when calculating errors for its radial profiles. Binned errors are then typically at the percent level (Figure 2). Except for the single central value for the force ratio in the BIMA northern core ( $\sim \pm 50 \%$ error $)$, similar errors are present in Figure 3 .

Finally, it is important to keep in mind that the correlation presented in Section 4.1 (based on one angle between two orientations) can be affected by projection effects. Generally, all values are integrated along the line of sight. The field significance $\Sigma_{B}$ presented here is much less or not at all affected by projection, because it is based on the ratio of two angles (Koch et al. 2012). It, nevertheless, still deals with quantities averaged along the line of sight. 


\section{Discussion}

\subsection{Self-Similar Profiles}

Given the common features in the radial profiles of the magnetic to gravitational force ratio (Figures 2 and 3), we address here the question of self-similarity. We focus on the JCMT main core where the BIMA and SMA data provide higher resolution follow-up observations. The BIMA southern core is adopted due to its better statistics. All three data sets in Figure 2 show a rather constant ratio across their cores (plateau-like or a single binned radius), followed by a plateau with a larger ratio before eventually the ratio grows to values larger than or around one. With the cores in the maps of Figure 1 being clearly identified, we choose to normalize the distances from the peaks to units of core sizes; i.e. all profiles are aligned to one normalized core size. The normalization radii, chosen to be those bins where the emission intensity profiles in Figure 2 flatten out, are 21."85, 3."42 and 1."06 for the JCMT, the BIMA and the SMA data, respectively. At these radii, the emissions are $\sim 15 \%$ to $20 \%$ of the peak emissions. Figure 4 shows the result.

Even with some uncertainty left in the normalization, it is obvious that the aligned profiles show a close resemblance. This self-similarity suggests that the interplay between magnetic and gravitational force is independent of the three different scales probed here. Thus, the analysis here quantifies the relative magnetic field significance from being generally dominant or comparable to gravity at distances twice the core size to minor or negligible inside the core. We stress that this result seems to hold generally for all the different field morphologies analyzed here, including even the more irregular cases shown in Figure 3 ,

\subsection{Mass-to-Flux Ratio Derived with Polarization Intensity-Gradient Method}

In this section we investigate the connection between the force ratio in Equation (2) and the mass-to-flux ratio for a molecular cloud. The mass scale associated with the amount of magnetic flux $\Phi$ threaded by a self-gravitating cloud was introduced in Mestel \& Spitzer (1956). The magnetic critical mass $M_{\Phi}=\frac{\Phi}{2 \pi G^{1 / 2}}$, with $\Phi=\pi R^{2} B$ where $R$ is the cloud radius, defines the maximum mass that can be supported by the magnetic field against gravitational collapse if no other forces are present (e.g. Shu et al. (1999)). This leads to the notions of magnetically supercritical clouds when $M>M_{\Phi}$, and magnetically subcritical clouds when $M<M_{\Phi}$. Contrary to the mass-to-flux ratio - which is typically applied to the entire (global) molecular cloud - the force ratio in Equation (2) is a local criterion comparing the field significance with gravity at a specific location in a cloud. Neglecting pressure gradients and explicitly writing out the local field force and gravity 
force terms, it can be expressed as a ratio of local flux over local mass:

$$
\frac{\sin \psi_{\ell}}{\sin \alpha_{\ell}}=\frac{\Phi_{\ell}^{2}}{m_{\ell}^{2}} \cdot \frac{m_{\ell}}{M_{\ell}} \cdot \frac{1}{R_{B, \ell}} \cdot \frac{1}{R_{\ell}^{4}} \cdot R_{G, \ell}^{2} \cdot \frac{1}{4 \pi^{3}} \frac{\eta}{G},
$$

where the lower index $\ell$ refers to a local quantity. $M_{\ell}$ is the gravitating mass leading to the local gravitational force $F_{G, \ell}=G \frac{m_{\ell} M_{\ell}}{R_{G, \ell}^{2}}$ acting upon a local mass element $m_{\ell}$ at a distance $R_{G, \ell}$. $\Phi_{\ell}$ is the flux associated with the local mass within a flux tube of radius $R_{\ell}$ (Figure 5). $R_{B, \ell}$ is the local field radius. $G$ and $\eta$ are the gravitational constant and a numerical unit conversion factor, respectively. Equation (3) is generally valid. However, in order to make further use of it, the local mass element $m_{\ell}$ and $M_{\ell}$ need to be specified. We, therefore, make the basic assumption of spherical symmetry. The enclosed mass within a distance $r$ from the center is then $M_{\ell}(r)=\int_{0}^{r} 4 \pi r^{\prime 2} \rho\left(r^{\prime}\right) d r^{\prime} \equiv N_{r} \bar{m}_{\ell}(r)$, where $N_{r}$ is the number of local flux tubes with an average local mass $\bar{m}_{\ell}(r)$ where both depend on the integration radius $r$. A local mass-to-flux ratio then results from Equation (3):

$$
\frac{m_{\ell}}{\phi_{\ell}}=\left(\frac{\sin \psi_{\ell}}{\sin \alpha_{\ell}}\right)^{-1 / 2} \cdot\left(\frac{m_{\ell}(r)}{\bar{m}_{\ell}(r)}\right)^{1 / 2} \cdot R_{B, \ell}^{-1 / 2} \cdot R_{\ell}^{-1} \cdot\left(4 \pi^{3}\right)^{-1 / 2} \cdot\left(\frac{\eta}{G}\right)^{1 / 2}
$$

where we have used $M_{\ell}(r)=N_{r} \bar{m}_{\ell}(r)$ with $N_{r}=\frac{R_{G, \ell}^{2}}{R_{\ell}^{2}}$. We note that the above expression is still fairly general and valid for any density profile $\rho(r)$. In particular, since the ratio depends on the ratio of the mass profile over a mean mass, only the functional form of the density profile is relevant, but not its absolute value. Introducing a mean local mass, $\bar{m}_{\ell}(r)$, allows us to write the local mass-to-flux ratio with the explicit spherical radius dependence only in the second term on the right hand side of Equation (4). Besides the spherical symmetry assumption for the mass distribution, Equation (4) is basically valid at any position in a molecular cloud5. The average inverse of the force ratio decreases with larger radius (Figure 4), and $\left(\frac{m_{\ell}(r)}{\bar{m}_{\ell}(r)}\right)^{1 / 2} \leq 1$ is monotonically decreasing for centrally peaked density profiles, with unity in the center. The local mass-to-flux ratio in Equation (4) is, thus, decreasing with larger radius from the center. Azimuthally averaging Equation (4) and normalizing it to the critical mass-to-flux ratio, we get the local normalized mass-to-flux ratio - for individual subvolumes of fixed size $R_{\ell}$ in a cloud - as a function of radius:

$$
\left(\frac{\Delta M}{\Delta \Phi}(r)\right)_{n o r m}=\left\langle\left(\frac{\sin \psi_{\ell}}{\sin \alpha_{\ell}}\right)^{-1 / 2} \cdot R_{B, \ell}^{-1 / 2}\right\rangle_{r} \cdot\left(\frac{m_{\ell}(r)}{\bar{m}_{\ell}(r)}\right)^{1 / 2} \cdot \frac{R_{\ell}^{-1}}{R_{0}^{-3 / 2}} \cdot \pi^{-1 / 2} \leq\left\langle\left(\frac{\sin \psi_{\ell}}{\sin \alpha_{\ell}}\right)^{-1 / 2}\right\rangle_{r} \cdot \pi^{-1 / 2}
$$

\footnotetext{
${ }^{5}$ We note that it is also possible to proceed directly with Equation (3). Assuming that both $m_{\ell}$ and $M_{\ell}$ are proportional to the integrated dust emission with an identical conversion factor, the ratio $\frac{m_{\ell}}{M_{\ell}}$ can directly be calculated from a dust continuum emission map. In this way, it is valid for any arbitrary cloud shape. This is identical to the approach outlined in Koch et al. (2012) where a local gravity direction is derived for any cloud shape. Equation (3), in its most general form, then leads to a map of local mass-to-flux ratios. However, we are here aiming at revealing changes in the mass-to-flux ratios with radius. Therefore, the spherical symmetry assumption and azimuthal averaging are appropriate (Equation (5)). In practice, we will calculate $\frac{m_{\ell}}{M_{\ell}}$ from the dust emission maps as mentioned above.
} 
where $\langle\ldots\rangle_{r}$ denotes azimuthal averaging at radius $r$. When normalizing to a local flux tube, we can simply set $R_{0} \equiv R_{\ell}$. Furthermore, as found in Koch et al. (2012), $R_{B, \ell}$ is roughly constant over an observed map, and similar to $R_{\ell}$, which we identify with the beam resolution of an observation. With this simplification, $\frac{R_{B, \ell}^{-1 / 2} R_{\ell}^{-1}}{R_{0}^{-3 / 2}} \sim 1$. Assuming the cloud gravitating mass to be proportional to the dust emission, $\frac{m_{\ell}}{M_{\ell}}$ is derived from the observed emission intensity profiles in Figure 2, As already indicated with the notation, Equation (5) quantifies the differential mass-to-flux ratio as a function of radius.

In a next step, we derive an integrated mass-to-flux ratio, i.e. we are asking whether the entire cloud inside a certain radius $r$ is magnetically supercritical. We then have to evaluate:

$$
\frac{M}{\Phi}(\leq r)=\frac{\sum^{N_{r}} m_{\ell}}{\sum^{N_{r}} \phi_{\ell}}=M_{\ell}(r) \cdot\left(\sum^{N_{r}} \phi_{\ell}\right)^{-1}
$$

$\phi_{\ell}$ can be expressed with Equation (4). The summation is over all local mass and flux elements within the radius $r$, where the summation limit $N_{r}$ depends on $r$. After some algebra and after normalizing with the critical mass-to-flux ratio we find:

$$
\left(\frac{M}{\Phi}(\leq r)\right)_{n o r m}=\left(\sum_{i=1}^{N_{b}}\left\langle\left(\frac{\sin \psi_{\ell}}{\sin \alpha_{\ell}}\right)^{1 / 2} \cdot R_{B, \ell}^{1 / 2}\right\rangle_{r_{i}} \cdot \frac{\bar{m}^{1 / 2}\left(r_{i}\right) \cdot m^{1 / 2}\left(r_{i}\right)}{\bar{m}(r)} \cdot N_{r_{i}}\right)^{-1} \cdot \frac{R_{l}^{-3}}{r^{-7 / 2}} \cdot \pi^{-1 / 2},
$$

where the averaging $\langle\ldots\rangle_{r_{i}}$ is for each bin $r_{i}$, with $N_{r_{i}}$ being the number of subvolumes within $r_{i}$ and $r_{i+1} . N_{b}$ is the number of bins within $r$ (Figure 5). Equation (7) states that the integrated mass-to-flux ratio is calculated by adding bin-averaged force ratios which are weighted with a mass function and $N_{r_{i}}$, where the latter one results from the integrated volume growing with $r$. The normalization is with respect to $r$, i.e. the increasingly larger cloud (with growing radius $r$ ) is normalized with its corresponding critical mass-to-flux ratio. $R_{\ell}$ is constant and again set by the beam resolution. $R_{B, \ell}$ is also roughly constant. When omitting the summation, setting $N_{r_{i}} \equiv 1$ and $r \equiv R_{\ell}$ and replacing $\bar{m}(r)$ with $\bar{m}\left(r_{i}\right)$, Equation (7) reduces to the differential mass-to-flux ratio in Equation (5).

Finally, a global mass-to-flux ratio for the entire cloud as a single entity can be derived from Equation (4) in the limiting case where all (small) local quantities grow to cloud-size: $m_{\ell} \rightarrow M$ and $\left\langle R_{B, \ell}>\approx<R_{\ell}>\approx<R_{G}>\equiv R\right.$. The force ratio needs to be averaged over the entire cloud: $\frac{\sin \psi_{\ell}}{\sin \alpha_{\ell}} \rightarrow\left\langle\frac{\sin \psi_{\ell}}{\sin \alpha_{\ell}}\right\rangle$. This then leads to the global mass-to-flux ratio, normalized to the critical massto-flux ratio with $R_{0}$ :

$$
\left(\frac{M}{\Phi}\right)_{n o r m}=\left\langle\left(\frac{\sin \psi_{\ell}}{\sin \alpha_{\ell}}\right)^{-1 / 2}\right\rangle \cdot\left(\frac{R}{R_{0}}\right)^{-3 / 2} \cdot \pi^{-1 / 2}
$$


where $R_{0} \equiv R$ is the cloud radius.

The set of Equations (5), (7) and (8) defines a differential, integrated and global mass-to-flux ratio, respectively, which can readily be calculated from observations. These equations provide the criteria to evaluate whether a subvolume (local or integrated up to a certain radius) or an entire cloud is magnetically supercritical. In all cases, the inverse square root of the force ratio is the key measure leading to an estimate independent of any mass or field strength input. Except for the global mass-to-flux ratio, the local ratios need the functional form of the mass profile as an additional input.

A schematic illustration of the different subvolumes analyzed by the various ratios is shown in Figure 5, Figure 6illustrates these mass-to-flux ratios for the SMA observation. The differential mass-to-flux ratio shows a clear transition from a magnetically subcritical state at larger radii to a supercritical state at smaller radii. This might indicate that a fixed subvolume can only collapse closer to the center, but not in the outer parts of a cloud/core. This finding possibly also provides an explanation for why fragmentation and clustering of stars preferentially occur at the center but not at larger radii. Such a result is further consistent with the theoretical work by Shu \& Li (1997), where the dilution of self-gravitational forces by the magnetic tension force (see Section 5.3 and Figure (4) is found to reduce the tendency of a disk to fragment. As further utilized in Section 5.3, this dilution is more significant at larger radii. Consequently, the fragmentation process is more significantly suppressed here. The number of individual collapsing subvolumes in the center region (with smaller or non-existent dilution) might then be linked to the multiplicity of stars. Additionally, the finding here might give support to the suggestion by Mouschovias (1976) that magnetic tension might be able to hold back the envelope and, therefore, prevent it from following the cloud core collapse. In a related later work, Shu et al. (2004) investigated whether magnetic tension can define the masses of forming stars by holding up the subcritical envelope of a molecular cloud while its supercritical core is collapsing. A split monopole - as it is possibly seen in the field morphology in the e 2 core (Tang et al. 2009b) - leads to magnetic levitation in their work. However, they find that magnetic suspension alone can not keep the subcritical envelope from falling onto the center, but additional dynamic levitation by winds and jets is needed for a halt of infall. The results here are also in qualitative agreement with the recent numerical simulation of giant molecular clouds with ambipolar diffusion by Vázquez-Semadeni et al. (2011). They find that the mass-to-flux ratio inside a cloud exhibits large spatial fluctuations, spanning an order of magnitude or more. Thus, local clumps can become magnetically supercritical within a globally subcritical system. Estimating $\mathcal{R}$ (the ratio between the mass-to-flux ratio in the core and in the envelope as introduced in Crutcher et al. (2009)) from the values at the largest distances and the values close to the center (Figure 6), we find $\mathcal{R}>1$. This would be in favor of ambipolar diffusion driven models. However, the recent statistical analysis of $\mathcal{R}$ based on numerical simulations with supersonic magnetohydrodynamical turbulence (Bertram et al. 2011) yields a large scatter in $\mathcal{R}$ with values 
both smaller and larger then unity. It, thus, has to be acknowledged that the interpretation of $\mathcal{R}$ as a measure to distinguish between ambipolar diffusion and turbulence driven star formation is possibly not unambiguous.

The integrated mass-to-flux ratio in Figure 6 is driven by the increasing volume. It, therefore, grows with radius. As expected, this ratio is generally larger than the differential ratio of a subvolume, because mass scales with volume whereas flux scales with area. The slight mismatch in the innermost bin results from different weightings for the two ratios. Finally, the global mass-to-flux ratio is close to the integrated ratio at large radii. The difference here results from the incomplete sampling of $N_{r_{i}}$ in azimuth for those bins which contain some depolarization zones. It is important to remark that both the integrated and global ratios present average values for the entire cloud. Therefore, they are likely biased toward some subvolumes. Whereas these ratios provide a description as to whether a cloud as an entity is in a sub- or supercritical state, they can not properly assess the state of individual subvolumes. This question can only be further addressed with the differential mass-to-flux ratio. The finding here clearly demonstrates the differences and the need for a measurement of the local field significance.

\subsection{Dynamical Implication and Star Formation Efficiency}

In this section we aim at investigating further implications of the local force ratio $\frac{\sin \psi}{\sin \alpha}$ (Figure (4) and the differential mass-to-flux ratio (Figure 6). We limit the discussion here to the collapsing core W51 e2. Global and/or average cloud properties are typically used to characterize its state; e.g. in order to determine whether a system is gravitationally bound, or whether it can collapse or not. With the local criteria - the magnetic field to gravity force ratio in Equation (2) and the differential mass-to-flux ratio in Equation (4) - we have tools to go one step further. We can not only address the question whether a cloud collapses or not, but we can even ask: Where does a cloud collapse? How does it collapse? Does all the gas take part in the collapse? And finally, depending on the answers to these questions, what are the consequences for the inferred star formation efficiency?

The change with radius in the magnetic field to gravity force ratio (Figure 4) clearly demonstrates that gravity is not everywhere equally efficient to initiate and to keep driving a collapse. The result in Figure 4 suggests and quantifies an effective gravitational force which is reduced by the presence of the magnetic field. Indeed, on the theory side, a concept of diluted gravity (by the

magnetic field) was put forward by Shu \& Li (1997). Thus, we can define an effectively acting gravitational force $F_{*}$ as

$$
F_{*}(r)=\left(1-\left\langle\frac{\sin \psi}{\sin \alpha}\right\rangle_{r}\right) \cdot F_{\max }(r) \equiv<\epsilon_{G}>_{r} \cdot F_{\max }(r),
$$

where $\langle\ldots\rangle_{r}$ denotes azimuthal averaging at radius $r$ and $F_{\max }(r)$ is the maximum non-diluted 
gravitational force resulting from an enclosed spherically symmetric mass distribution within $r . F_{*}$ is only defined where $\frac{\sin \psi(r)}{\sin \alpha(r)} \leq 1$, which possibly holds only for a limited area. In Equation (9) we have introduced the gravity efficiency factor $\epsilon_{G} \in[0,1] 6$.

Besides the diluted gravity which slows down the accretion and collapse process, the fraction of volume of a cloud (or core) that actually can collapse is relevant; i.e. where is the cloud/core magnetically supercritical? The differential mass-to-flux ratio (Figure 6) provides a local criterion that answers this question. The increasing ratio toward the center indicates that only the central part of the core has accumulated enough mass to overcome the magnetic flux. By reading off the radius where the core state changes from magnetically sub- to supercritical, the potentially collapsing volume and its associated mass can be estimated. This defines a volume efficiency, $\epsilon_{V} \in[0,1]$, as compared to the maximum efficiency $\epsilon_{V} \equiv 1$ where the entire core can collapse.

Based on the two above described findings, we proceed to estimate a star formation efficiency with the assumptions: (1) the available effective gravitational force is diluted by the presence of the magnetic field and (2) only the regions where the differential mass-to-flux ratio is larger than one will eventually collapse and form stars. We choose to reference our estimate to the well-known pressure-less free-fall collapse of a spherical gas sphere. The dynamics in this case are governed by the momentum equation $\frac{D v}{D t}=-G \frac{M_{r}}{r^{2}}$, where the convective derivative is $\frac{D v}{D t}=\frac{\partial v}{\partial t}+v \frac{\partial v}{\partial r}$ for the gas infall velocity $v$. The enclosed mass within radius $r$ is $M_{r}=\int_{o}^{r} 4 \pi r^{\prime 2} \rho\left(r^{\prime}\right) d r^{\prime}$ with the gas density $\rho$. With the diluted gravity, the momentum equation reads:

$$
\frac{D v}{D t}=-<\epsilon_{G}>_{r} \cdot \frac{G M_{r}}{r^{2}} .
$$

Technically, the gravity efficiency factor $\left\langle\epsilon_{G}>_{r}\right.$ could be absorbed into an effective density $\rho_{*}(r)$. Therefore, the general solution (for any $M_{r}$ ) of the Equation (10) for a fluid element starting at a distance $r_{0}$ and reaching the center at $r=0$ at the time $t$ is still valid. The time $t_{*}$ in the presence of the magnetic field is then 7 :

$$
t_{*}=\frac{\pi}{2} \sqrt{\frac{r_{0}^{3}}{2 G M_{r}\left(\rho(r),<\epsilon_{G}>_{r}\right)}} \approx<\epsilon_{G}>^{-1 / 2} \cdot t
$$

\footnotetext{
${ }^{6}$ Unlike the gravity efficiency factor $\epsilon_{G}$, the field significance $\Sigma_{B}$ in Equation (2) is not constrained to an upper limit of one.

${ }^{7}$ For a uniform density and $<\epsilon_{G}>_{r} \equiv 1$, Equation 111) describes a synchronized collapse with the well-known free-fall time $t_{f f}=\sqrt{\frac{3 \pi}{32 G \rho}}$. More realistic density profiles with a central concentration, e.g. $\rho(r)=\rho_{c}\left(\frac{r}{r_{c}}\right)^{-\alpha}$ lead to a collapse time $t=\sqrt{\frac{(3-\alpha) \pi}{32 G \rho_{c}}} \cdot\left(\frac{r_{0}}{r_{c}}\right)^{\alpha / 2}$ which increases with larger distance $r_{0}$ from the center and then reproduces an inside-out collapse.
} 
Strictly speaking, $\left\langle\epsilon_{G}\right\rangle_{r}$ in the above equation is still a function of radius. In writing the right hand side we have assumed it to be averaged within an appropriate radius. The average accretion rate $\langle\dot{M}>$, assuming the entire mass $M$ within a cloud/core to be accreted and to collapse, is $<\dot{M}>=M / t$. Adding the volume efficiency factor $\epsilon_{V}$ together with Equation (11), the effective accretion rate $\left\langle\dot{M}_{*}\right\rangle$, corrected for our observed magnetic field features, becomes:

$$
<\dot{M}_{*}>=\frac{M_{*}}{t_{*}} \approx \frac{\epsilon_{V} \cdot M}{<\epsilon_{G}>^{-1 / 2} \cdot t} .
$$

The resulting star formation efficiency relative to the standard free-fall one is then expressed as:

$$
\frac{\left\langle\dot{M}_{*}>\right.}{\langle\dot{M}>} \approx \epsilon_{V} \cdot\left\langle\epsilon_{G}>^{1 / 2} .\right.
$$

We note that the above phenomenological modeling is still valid in the presence of additional force terms in the momentum equation with any density profiles. The pressure-less free-fall collapse simply allows us to analytically express the results. Figure 7 shows the reduced star formation efficiency taking into account the effects of the magnetic field. The red dots indicate efficiencies estimated from the gravity dilution and the reduced collapse volume based on the Figures 4 and 6. Since $1-\frac{\sin \psi(r)}{\sin \alpha(r)} \sim 0$ at large distances, an arbitrarily low star formation efficiency would result from adopting these values for $\epsilon_{G}$. Instead, we adopt values averaged over the entire core (force ratio $\sim 0.33)$, the central core only $(\sim 0.2)$ and the outer plateau $(\sim 0.85)$. We consider these values adequate to estimate and sample the gravity dilution for W51 e2. Similarly, for the volume efficiency factor we read a radius of about $1^{\prime \prime}$ within which the core is magnetically supercritical as compared to an overall size of about $2^{\prime \prime}$. For comparison, a larger volume of about $1.5^{\prime \prime}$ is considered as well. The resulting efficiencies are reduced at least to a conservative value of $\sim 0.35$ or less when still assuming the larger volume to collapse. The efficiencies drop to $\sim 0.1$ with the smaller volume, with a likely limit of about $\sim 0.05$ in combination with the largest observed gravity dilution. These values are close to the observationally inferred efficiencies of a few percent (e.g., Krumholz \& Tan 2007). For comparison, recent numerical simulations by Nakamura \& Li (2011) find a star formation efficiency per global free-fall time of $\sim 20$ to $\sim 30 \%$ depending on the magnetic field strength. When they further take into account the feedback from outflows, their efficiencies can be reduced by another order of magnitude.

We finally remark that the discussion here is limited to the core e2. A more complete picture, estimating the star formation efficiency starting from the largest scales of the cloud envelop, will need to dynamically link the observed self-similar profiles from Figure 4. Caution is needed here because linking structures of different size scales is non-trivial due to additional structures possibly generated by, e.g. fragmentation and turbulence. Nevertheless, this first estimate further solidifies the significance and impact of the magnetic field. Moreover, it demonstrates that the magnetic field is capable of reducing the star formation efficiency by at least one order of magnitude or even more. 


\section{Summary and Conclusion}

We have applied the polarization - intensity gradient method (Koch et al. 2012) to a set of single dish and interferometer dust continuum data of the W51 star formation region. These observations cover scales from the initial parent cloud $(\sim 2 \mathrm{pc}, \mathrm{JCMT})$ to the large-scale envelope $(\sim$ $0.3 \mathrm{pc}, \mathrm{BIMA})$ and to the collapsing core $(\sim 60 \mathrm{mpc}, \mathrm{SMA})$. Besides leading to a magnetic field strength as a function of position in a map, our new method also provides a way to estimate the local magnetic field to gravity force ratio. The technique is model-independent, without any input from mass or field strength, solely making use of measured angles in dust polarization and Stokes I map. Here, we have focused on this force ratio and on some of its implications. Our main results are summarized in the following.

1. Magnetic field - intensity gradient correlation: The correlation in the magnetic field and dust intensity gradient orientations - which served as a starting point for the new method developed in Koch et al. (2012) - is also found at larger scales in the BIMA and JCMT data. The correlation coefficients seem to be the larger the higher the resolution is; i.e. the tightest correlation is found for the collapsing core e2 (Figure 1, left panels).

2. Magnetic Field Significance: Maps of the magnetic field to gravity force ratio show distinct features for all three data sets (Figure 1, right panels). At larger distances from the emission peaks, the field force is comparable or larger than the gravity force (ratio $\gtrsim 1$ ). Closer to the emission peaks, gravity becomes more dominant (ratio $<1$ ). This suggests and quantifies that gravity is not everywhere acting equally efficiently, but is being more significantly opposed by the magnetic field tension force at larger distances. Based on this finding, we introduce a gravity efficiency factor. This also seems to be in agreement with the concept of gravity dilution (Figure 4). Moreover, this is in support of inside-out collapse scenarios.

3. Self-similarity: Azimuthally averaged radial profiles of the force ratios show similar features for the JCMT, BIMA and the SMA data (Figure 2). When normalized to core sizes, these profiles closely align (Figure 4). This points toward self-similar properties in the magnetic field and gravity interplay from the large parent cloud down to the collapsing core.

4. Mass-to-flux ratio: The force ratio can be converted into a mass-to-flux ratio. In its most general form, the mass-to-flux ratio can then be expressed as the inverse square root of the force ratio multiplied by the square root of a ratio of two mass elements. With the force ratio being a local criterion, this naturally leads to a local (differential) mass-to-flux ratio for a subvolume in a molecular cloud as a function of position. An integrated and global massto-flux ratio can be derived by appropriately adding and averaging local quantities. Similar to the force ratio, all the various mass-to-flux ratios do not have to rely on absolute mass 
and field strength inputs, but they are largely model-independent. This finding then does not only provide a criterion to decide whether a molecular cloud as an entity is magnetically supercritical or not, but it extends this criterion to any subvolume (Figures 5 and 6). In the case of W51 e2, a transition occurs from subcritical at larger distances to supercritical in the central area. This might explain why fragmentation is found to happen preferentially in the center.

5. Starformation efficiency: A reduced star formation efficiency (compared to the non-magnetized free-fall case) is derived based on two observed magnetic field properties: (1) Gravity dilution (Figure 4) defines an effectively available gravity force to initiate and drive a collapse and, therefore, it increases the collapse time. (2) The differential mass-to-flux ratio (as a function of radius) provides a hint that the magnetic field reduces the volume of supercritical state and, therefore, only a limited volume can collapse and form stars (Figure 6). With these two findings a modified accretion rate can be calculated. Comparing this to a standard free-fall rate shows that the magnetic field is capable of reducing a standard star formation efficiency to $10 \%$ or less.

The authors thank the referee for valuable comments which led to further important insight in this work. P.T.P.H. is supported by NSC grant NSC97-2112-M-001-007-MY3.

\section{REFERENCES}

Allen, A., Li, Z.-Y., \& Shu, F. H. 2003, ApJ, 599, 363

Bertram, E., Federrath, C., Banerjee, R., \& Klessen, R.S. 2011, arXiv:1111.5539v1

Chrysostomou, A., Aitken, D.K., Jenness, T., et al. 2002, A\&A, 385, 1014

Crutcher, R.M., Hakobian, N., \& Troland, T.H. 2009, ApJ, 692, 844

Dotson, J.L., Vaillancourt, J.E., Kirby, L., et al. 2010, ApJS, 186, 406

Draine, B.T., \& Weingartner, J.C. 1996, ApJ, 470, 551

Draine, B.T., \& Weingartner, J.C. 1997, ApJ, 480, 633

Falceta-Gonçalves, D., Lazarian, A., \& Kowal, G. 2008, ApJ, 679, 537

Genzel, R., Downes, D., Schneps, M.H., et al. 1981, ApJ, 247, 1039

Girart, J.M., Rao, R., \& Marrone, D.P. 2006, Science, 313, 812 
Girart, J.M., Beltrán, M. T., Zhang, Q., Rao, R., \& Estalella, R. 2009, Science, 324, 1408

Hezareh, T., Houde, M., McCoey, C., \& Li, H. 2011, arXiv:1007.2242

Hildebrand, R.H. 1988, QJRAS, 29, 327

Hildebrand, R.H., Kirby, L., Dotson, J.L., Houde, M., Vaillancourt, J.E. 2009, ApJ, 696, 567

Ho, P.T.P., \& Young, L.M. 1996, ApJ, 472, 742

Ho, P.T.P., Moran, J.M., \& Lo, K.-Y. 2004, ApJ, 616, 1

Houde, M., Vaillancourt, J.E., Hildebrand, R.H., Chitsazzadeh, S., \& Kirby, L. 2009, ApJ, 706, 1504

Koch, P.M., Tang, Y.-W., \& Ho, P.T.P. 2010, ApJ, 721, 815

Koch, P.M., Tang, Y.-W., \& Ho, P.T.P. 2012, ApJ, accepted

Krumholz, M. R. \& Tan, J. C., 2007, ApJ, 656, 959

Lai, S.-P., Crutcher, R.M., Girart, J.M., \& Rao, R. 2001, ApJ, 561, 864L

Li, H., \& Houde, M. 2008, ApJ, 677, 1151

Li, Z.-Y., \& Nakamura, F. 2004, ApJ, 609, L83

Mestel, L., \& Spitzer, L. 1956, MNRAS, 116, 505

Nakamura, F., \& Li, Z.-Y. 2011, arXiv:1107.3616

Lazarian, A. 2000, ASPC, 215, 69

Mouschovias, T.Ch. 1976, ApJ, 207, 141

Rao, R., Girart, J.M., Marrone, D.P., Lai, S.-P., \& Schnee, S. 2009, ApJ, 707, 921

Rudolph, A., Welch, W. J., Palmer, P., \& Dubrulle, B. 1990, ApJ, 363, 528

Sollins, P. K., Zhang, Q., \& Ho, P.T.P., 2004, ApJ, 606, 943

Surcis, G., Vlemmings, W.H.T., Curiel, S., et al. 2011, A\&A, 527A, 48S

Shi, H., Zhao, J.-H., \& Han, J.L. 2010, ApJ, 718, L181

Shu, F.H., \& Li, Z.-Y. 1997, ApJ, 475, 251 
Shu, F.H., Allen, A., Shang, H., Ostriker, E.C. \& Li, Z.-Y. 1999, in The Origin of Stars and Planetary Systems, edited by Charles J. Lada and Nikolaos D. Kylafis, Kluwer Academic Publishers, 1999, p.193

Shu, F.H., Li, Z.-Y., \& Allen, A. 2004, ApJ, 601, 930

Tang, Y.-W., Ho, P.T.P., Girart, J.M., et al. 2009a, ApJ, 695, 1399

Tang, Y.-W., Ho, P.T.P., Koch, P.M., et al. 2009b, ApJ, 700, 251

Tang, Y.-W., Ho, P.T.P., Koch, P.M., \& Rao, R. 2010, ApJ, 717, 1262

Vázquez-Semadeni, E., Banerjee, R., Gómez, G.C., et al. 2011, MNRAS, 414, 2511

Vlemmings, W.H.T., Humphreys, E.M.L., \& Franco-Hernández, R. 2011, ApJ, 728, 149

Young, L. M., Keto, E., \& Ho, P.T.P., 1998, ApJ, 507, 270

Zhang, Q., \& Ho, P.T.P., 1997, ApJ, 488, 241

Zhang, Q., Ho, P.T.P., \& Ohashi, H., 1998, ApJ, 494, 636 

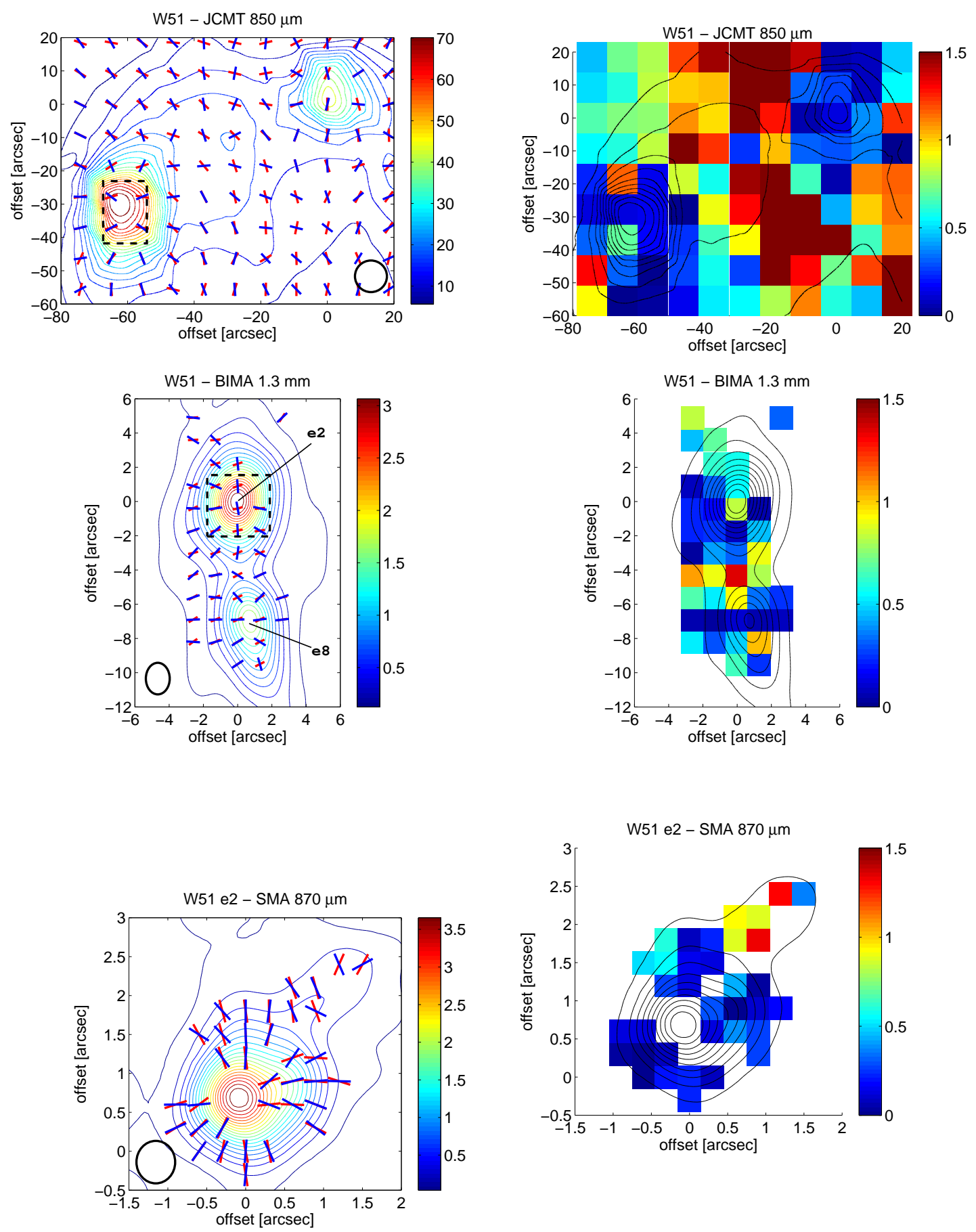

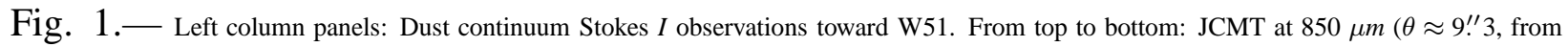

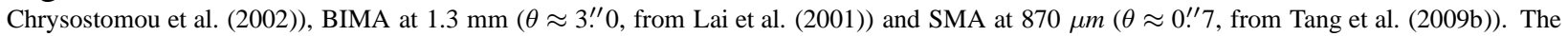
beam resolutions are shown with the black ellipses in the lower left or right corners. The BIMA observation is zooming in onto the main core (dashed rectangle) in the JCMT map. The BIMA main core (dashed square) is further resolved in the SMA map. The cores e2 and e8 are labeled. Colors correspond to the color wedges on the right hand side with units in Jy/beam. Overlaid are the magnetic field segments (red segments) and the intensity gradient segments (blue segments) at the locations where polarized emission was detected. Magnetic field segments are plotted by rotating the polarization segments by $90^{\circ}$. The difference in P.A.s between the magnetic field and the intensity gradient is the angle $\delta$ (Section 4.1. The length of the segments is arbitrary, and for visual guidance only. The axes offset positions (in arcsec) are with respect to the original (phase) centers of the observations. At the distance of W51, $1^{\prime \prime}$ is equivalent to about $30 \mathrm{mpc}$. Right column panels: The ratio of the magnetic field force $F_{B}$ compared to the gravitational force $F_{G}, \Sigma_{B}=\frac{\sin \psi}{\sin \alpha}$, as a function of position. The pixel scale is identical to the gridding in the left column panels. From top to bottom are shown the maps for the JCMT, BIMA and the SMA observations. Overlaid in black are the Stokes $I$ dust continuum emission contours from Figure 1 Colors correspond to the color wedges on the right hand side, displaying the dimensionless ratio. Blue colors indicate where gravity dominates over the magnetic field $\left(F_{B}<F_{G}\right)$. 

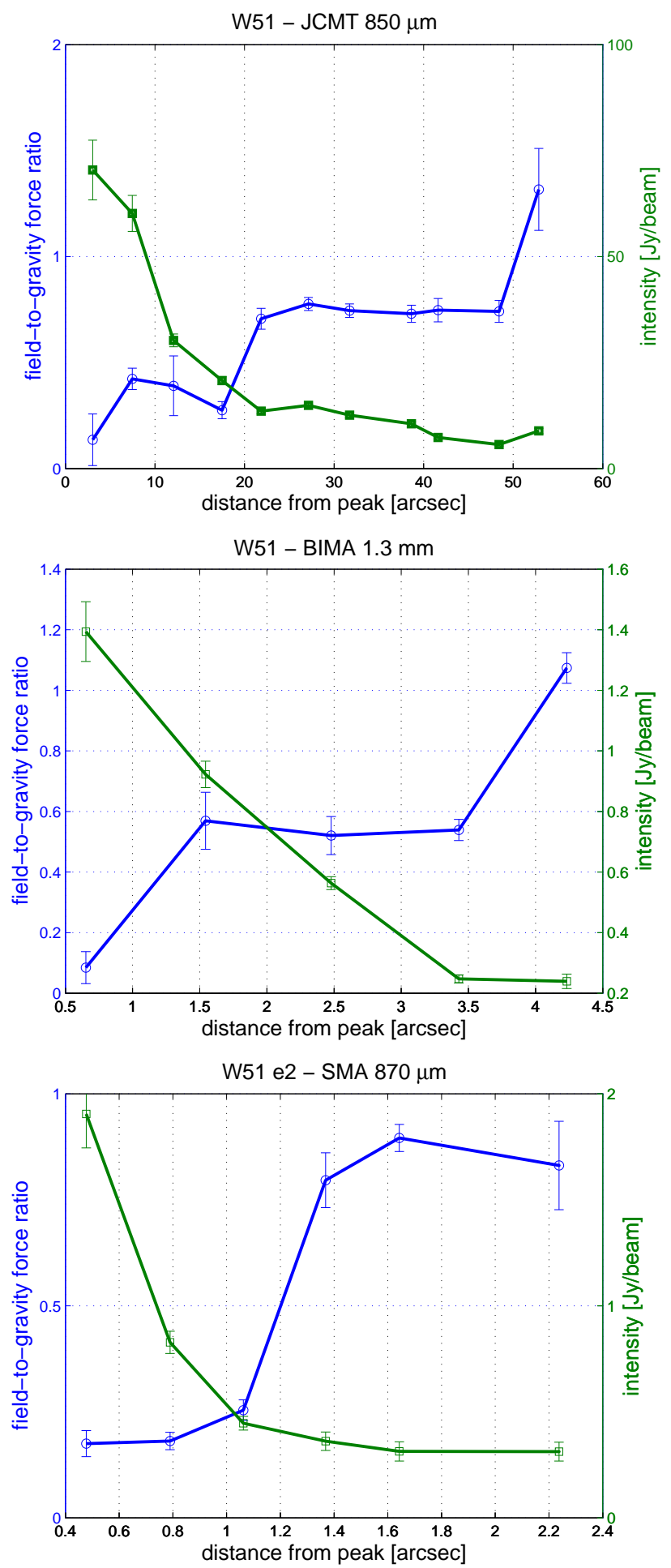

Fig. 2. - The magnetic field to gravitational force ratio, $\Sigma_{B}=\frac{\sin \psi}{\sin \alpha}$, as a function of distance from the emission peaks (blue). From top to bottom shown are the JCMT, BIMA and the SMA results. The corresponding intensity emission profiles are displayed in green with the axis at the right hand side. Values are azimuthally averaged and binned at half of the beam resolution. From the JCMT and BIMA observations, the results for the main peak and the southern peak, respectively, are shown. Binned values are connected with lines for visual guidance only. Errors for the force ratios are calculated by propagating typical measurement uncertainties in $\psi$ and $\alpha$ through Equation [2]. A 10\% uncertainty in the intensity emission is assumed. Error bars at the smallest and largest scales are typically growing because of a smaller sample variance factor. Binned errors are all around $\sim \pm 10 \%$ or less, with the only exception being the JCMT data at the largest scale with an error of $\sim \pm 20 \%$. 

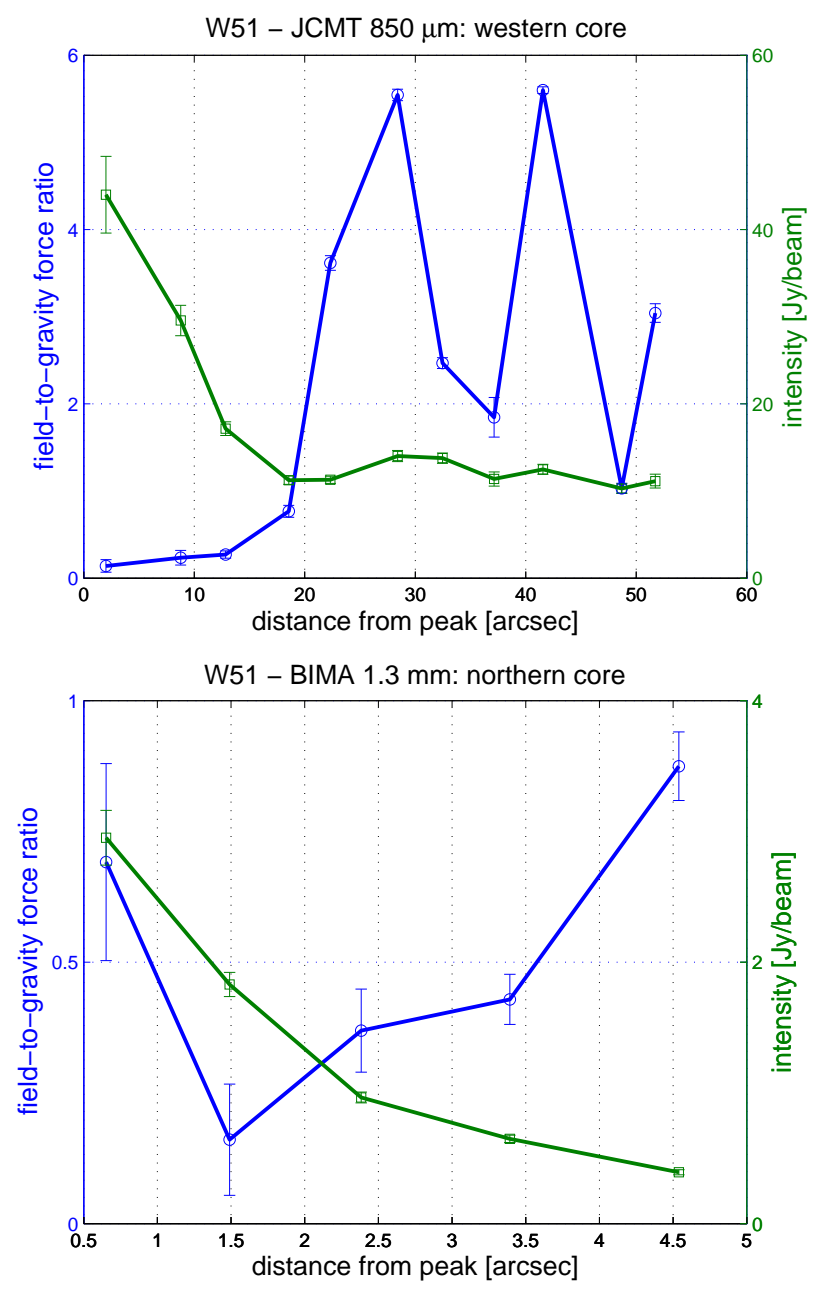

Fig. 3.- As in Figure 2, the magnetic field to gravitational force ratio with the corresponding intensity emission profile as a function of distance from the emission peak for the JCMT western and the BIMA northern core. 


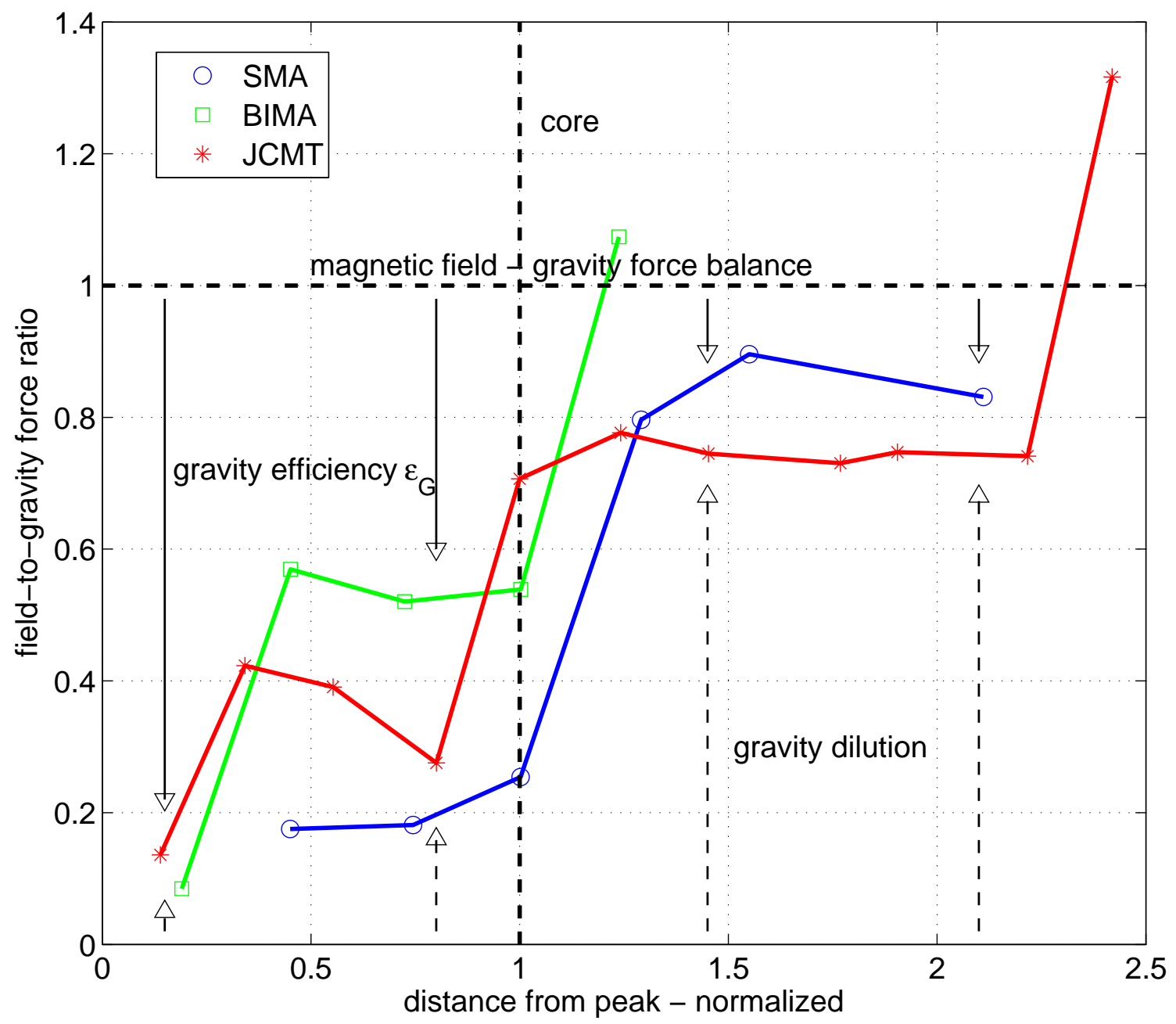

Fig. 4.- The magnetic field to gravitational force ratio, as in Figure 2, but with the distances from the peaks normalized to units of the core sizes. With respect to Figure 2 , the normalization radii are 21".85, 3."42 and 1."06 for the JCMT, the BIMA and the SMA data, respectively. The force balance $\left(\frac{\sin \psi}{\sin \alpha}=\Sigma_{B} \equiv 1\right)$ is indicated with the horizontal dashed black line. The effectively acting gravitational force (as introduced in Equation (9)) is derived with the gravity efficiency $\left\langle\epsilon_{G}>_{r}=1-\left\langle\frac{\sin \psi}{\sin \alpha}\right\rangle_{r}\right.$, which is symbolized through the lengths of the down-arrows. Similarly, the gravity dilution (by the magnetic field) is indicated with the dashed up-arrows. The error bars, identical to the ones in Figure 2, are omitted here. 


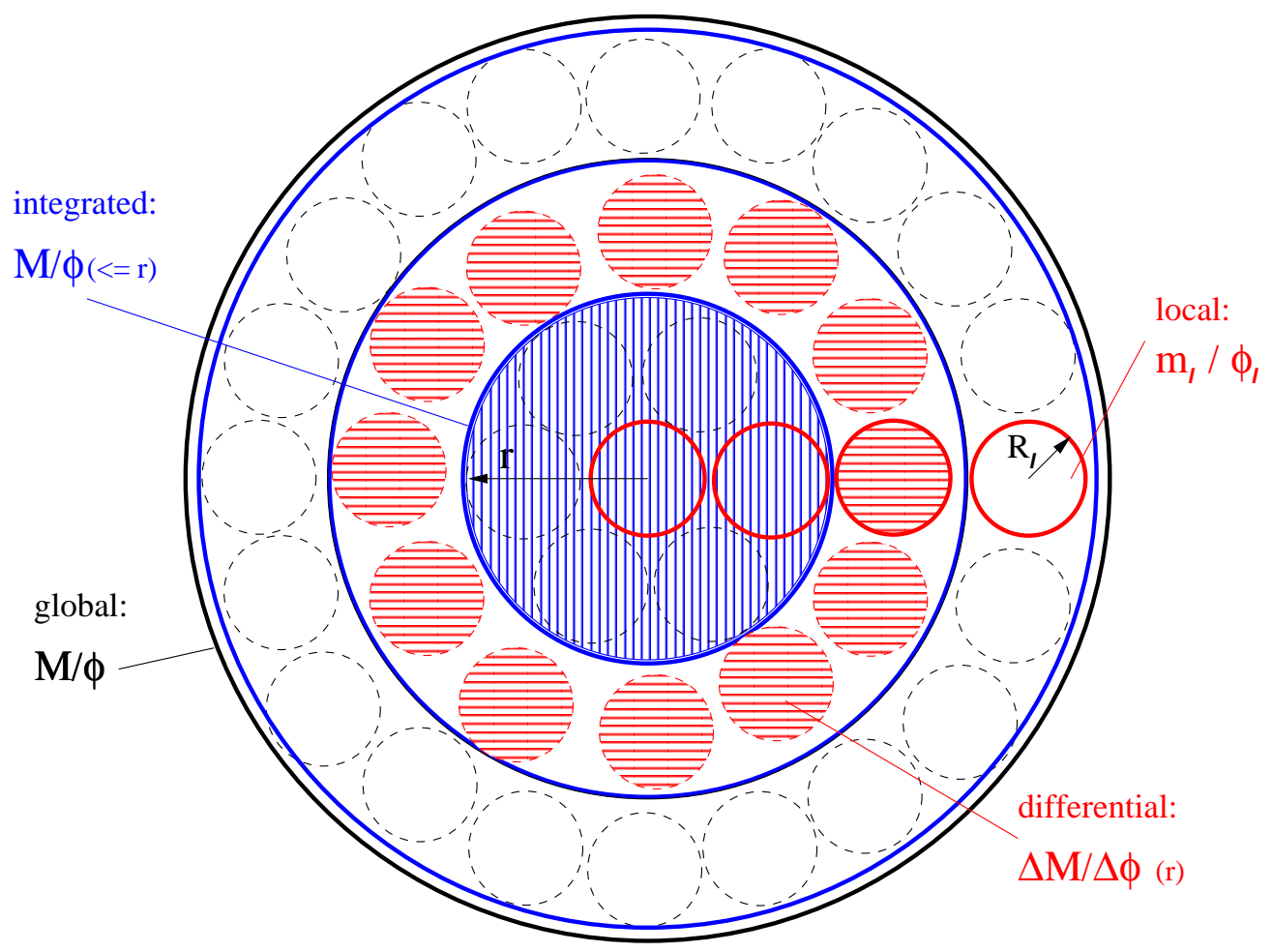

Fig. 5.- Illustration of the various mass-to-flux ratios as derived in Section 5.2, Every small circle of radius $R_{\ell}$ represents a local mass-to-flux ratio $\left(\frac{m_{\ell}}{\phi_{\ell}}\right.$, Equation (4)). Its size is determined by the beam resolution. The differential mass-to-flux ratio $\left(\frac{\Delta M}{\Delta \Phi}(r)\right.$, Equation (5) $)$ at radius $r$ is calculated by azimuthally averaging all the local ratios of the hatched red circles inside the ring at radius $r$. The four solid red circles symbolize differential ratios at four different radii. The integrated mass-to-flux ratio $\left(\frac{M}{\phi}(\leq r)\right.$, Equation (7) $)$ within radius $r$ is shown with the blue hatched circle. Concentric blue circles with growing radii symbolize integrated ratios for increasingly larger volumes of the cloud. Azimuthally averaged ratios for each radius are displayed in the profiles in Figure 6. A single global ratio $\left(\frac{M}{\phi}\right.$, Equation (8) $)$ is calculated from the black circle encompassing the entire cloud. 


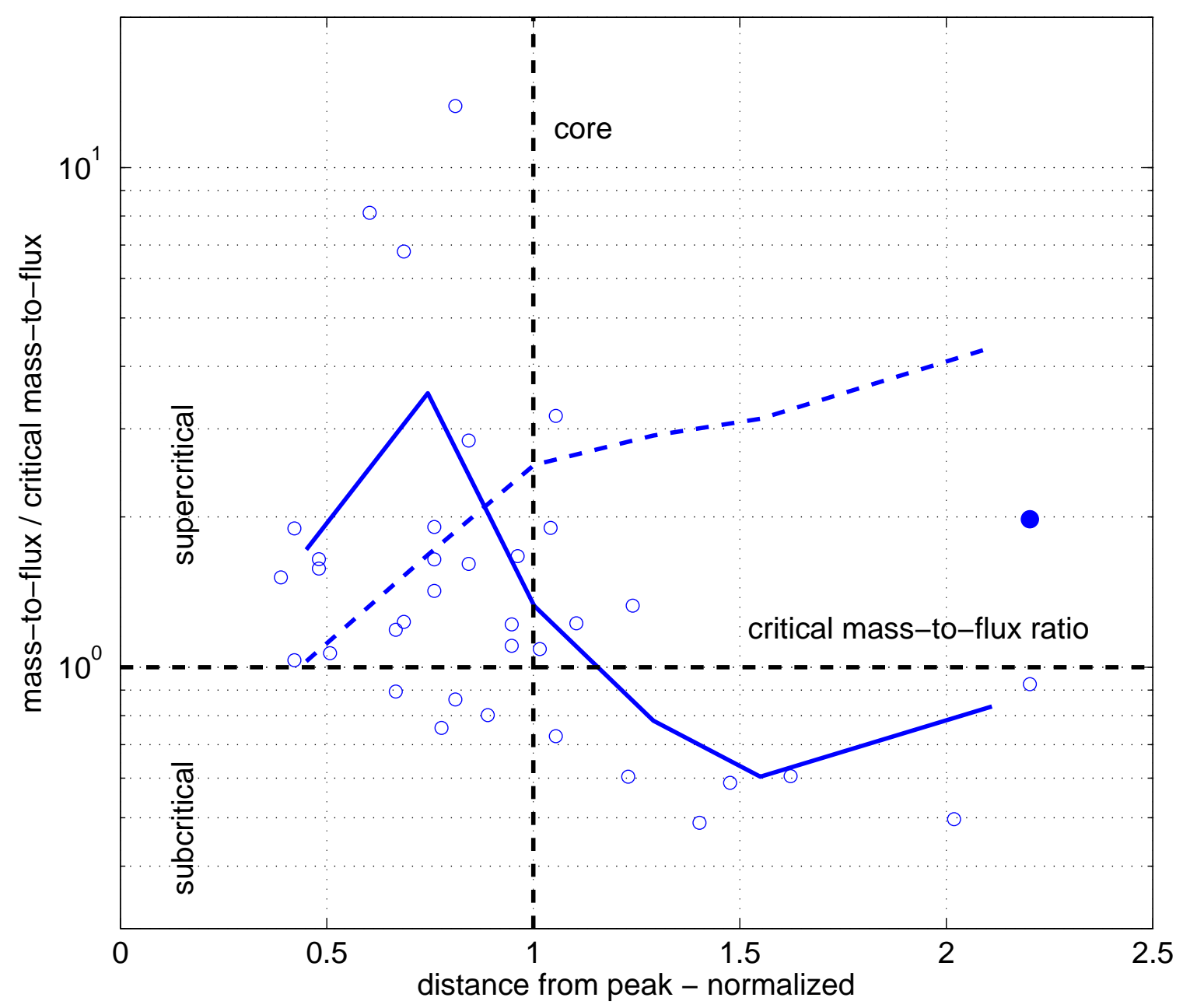

Fig. 6. - The various mass-to-flux ratios normalized to the corresponding critical mass-to-flux ratios (Section 5.2) for W51 e2. The ratios are shown as a function of distance from the peak normalized to the core size. The solid line displays the normalized azimuthally averaged differential mass-to-flux ratios (Equation (5), converted from the magnetic field to gravitational force ratios (Figure 2) with the additional mass ratio, $\left(\frac{m_{\ell}(r)}{\bar{m}_{\ell}(r)}\right)^{1 / 2} \leq 1$. The mass ratio is derived from the integrated emission profiles in Figure 2. A clear transition between magnetically supercritical in the center to subcritical at larger distances is revealed. Open circles correspond to the individual ratios before averaging and before multiplying with the mass ratio. Thus, they are local upper limits (Equation (5)). The dashed line is the integrated mass-to-flux ratio (Equation (7)) normalized to the critical mass-to-flux ratio with its radius corresponding to each binning radius. The single global mass-to-flux ratio (Equation $(8)$ ), averaging all force ratios and normalizing to the core radius, is displayed with the filled circle. Indicated with black dashed lines are the critical mass-to-flux ratio and the core size (normalized to one). When propagating uncertainties through $\frac{\Delta M}{\Delta \Phi} \sim\left(\frac{\sin \psi}{\sin \alpha}\right)^{-1 / 2}$, errors are suspect to an additional factor $\frac{1}{2}\left(\frac{\sin \psi}{\sin \alpha}\right)^{-3 / 2}$ as compared to the force ratios (Figure 2). Thus, at larger radii where the force ratio is around one, errors in the mass-to-flux ratios are similar to or smaller than the ones for the field-to-gravity force, i.e. $\lesssim \pm 10 \%$. As the force ratios drop in the center area, errors grow larger by a factor of about 2 to 3 , i.e. reaching $\sim \pm 20 \%$ to $\sim \pm 30 \%$. For clarity, error bars are not displayed here. 

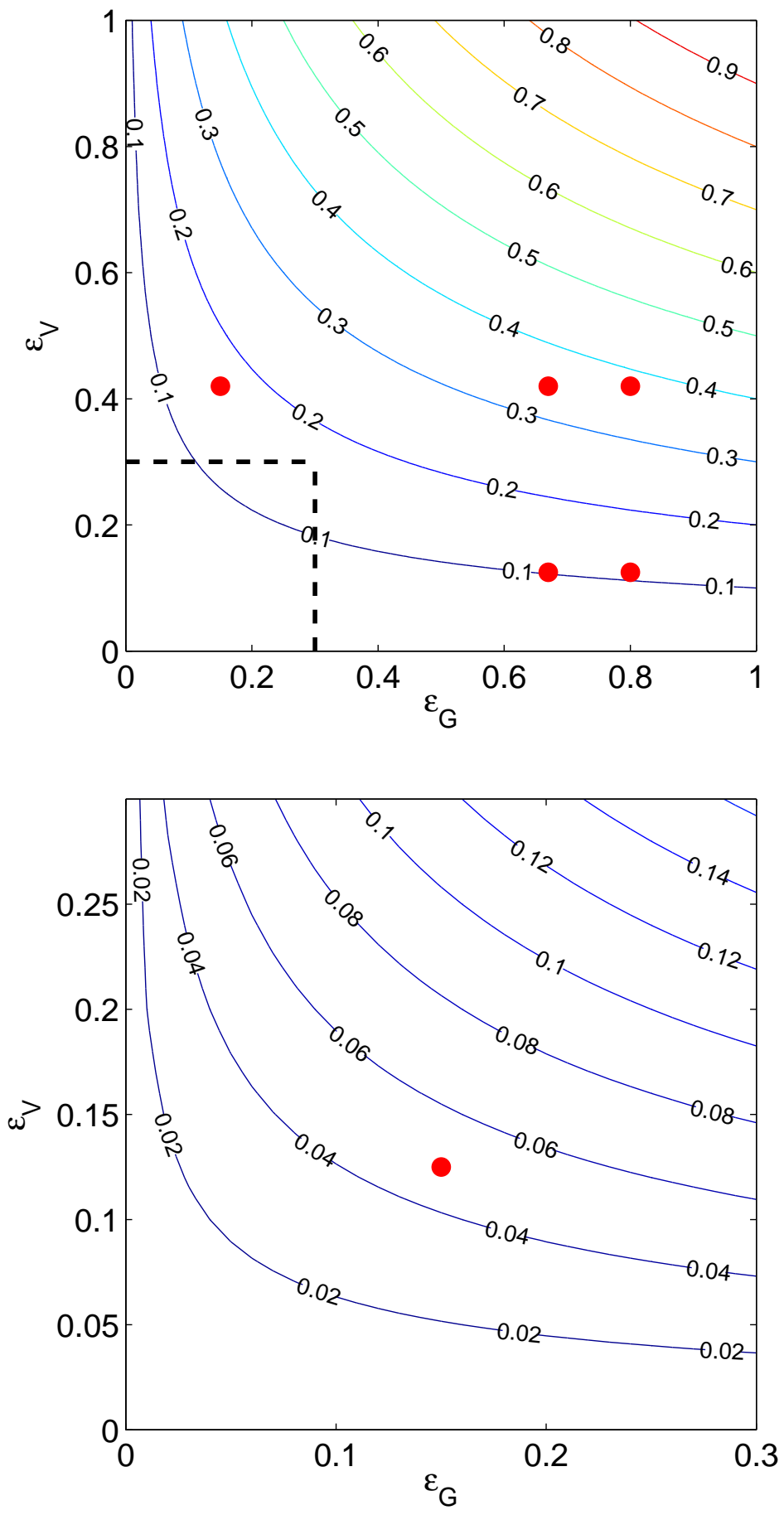

Fig. 7.- Estimated reduced star formation efficiency (contour levels) with a diluted gravitational force (efficiency factor $\epsilon_{G}$ ) and a reduced collapsing volume (efficiency factor $\epsilon_{V}$ ) following Equation (13). The lower panel shows the zoomed-in area marked with dashed lines in the upper panel. The red dots are possible estimated efficiencies based on the force ratio (Figure 4) and the differential mass-to-flux ratio (Figure 6) for the case of W51 e2. 